\title{
RICARDO PALMA Y LA LENGUA INGLESA'
}

\section{Ricardo Palma and the English language}

\section{ClAUdia ISABEL ZEgARRA DEL CASTILLO}

Facultad de Ciencias Económicas y Empresariales, Universidad Ricardo Palma Lima, Perú

Contacto: claudia.zegarra@urp.edu.pe

\section{RESUMEN}

Siguiendo el espíritu de reflexión del patriarca de las letras, este artículo tiene como objetivo dar a conocer ciertos aspectos de la vida de Ricardo Palma como traductor y conocedor de la obra de los literatos más representativos del Romanticismo europeo y americano. Diversos autores y numerosas fuentes dan cuenta de un primer contacto de Palma con escritores destacados de habla inglesa, que se cree impelieron al escritor a dedicar muchas horas robadas al descanso para traducir, producir sus propios versos y perfeccionar el estilo literario con el que consagraría la gran evolución intelectual de sus obras. Es menester del traductor explorar la biografía de todo escritor para desvelar los mensajes ocultos entre su pluma y sus páginas, ya que conocer la estrecha relación

1 Este artículo se elaboró como parte del curso Taller de Comunicación Oral y Escrita II, y tuvo como asesores al Dr. Carlos Pérez Garay y a la Lic. Melissa Sabino Parra. 
que mantuvo Palma a lo largo de los años con la cultura inglesa podría acercarnos al verdadero espíritu de su pasión por las letras.

Palabras clave: Ricardo Palma; traducción literaria; lengua inglesa.

\section{ABSTRACT}

Following the spirit of reflection of the patriarch of letters, this article aims to show certain aspects of Ricardo Palma's life as a translator and expert on the work of the most representative literary figures of European and American Romanticism. Several authors and numerous sources show Palma's first contact with outstanding English-speaking writers, who are believed to have encouraged the writer to dedicate many hours stolen from his free time to translating, producing his own verses and perfecting the literary style with which he would consecrate the great intellectual evolution of his works. It is the translator's duty to explore the biography of every writer to discover the hidden messages between his pen and his pages, as knowing the close relationship that Palma maintained over the years with English culture could bring us closer to the true spirit of his passion for literature.

Key words: Ricardo Palma; literary translation; English language.

Recibido: 08/11/2019 Aceptado: 08/12/2019 


\section{INTRODUCCIÓN}

Al repasar la vida y obra del notable escritor tradicionalista Ricardo Palma, una serie de cuestionamientos florece en nuestro pensamiento, atrayéndonos hacia la búsqueda de aquello que le sirvió de inspiración, de sus vivencias más preponderantes e inéditas, y de un íntimo acercamiento a quienes influyeron en su forma de pensar, escribir y crear, hasta hacerlo capaz de confluir en un estilo literario auténtico, propio y completamente nuevo para la literatura peruana del siglo XIX.

¿Cómo nos acercamos a una primera interpretación de su exquisita genialidad creativa? En la búsqueda de este conocimiento intrínseco del autor, surge la necesidad de partir por su formación, sus primeros años como escritor, así como de su primer contacto con la cultura occidental, de quien devino su faceta más romántica y emocional: la de traductor de poemas. En las siguientes páginas, revisaremos aquellos lugares que visitó, así como aquellas personas que conoció y que podrían haber constituido el pilar de las creaciones más bellas de su espíritu.

\section{SUS PRIMEROS AÑOS}

Ricardo Palma fue un escritor nato. Se valió de una respetable formación literaria en el emblemático Convictorio de San Carlos, donde concibió la disciplina y el orden, el método y el estilo, pero sobre todo la certeza de convertirse en un verdadero catón de las letras.

Palma era todavía un niño cuando recibió clases de francés en el colegio Clemente Noel, donde, hacia octubre de 1845, ocupaba el octavo lugar en el curso completo, demostraba su afición por los idiomas, y fue presentado como alumno destacado en los exámenes públicos ofrecidos a fines de 1846 (Holguín, 1995, pp. 112-113). 
Con apenas quince años, en el año 1848, Palma formó parte, junto con otros jóvenes apasionados, de un grupo romántico fuertemente influenciado por la cultura europea de aquel tiempo, al que bautizó como «Los Bohemios» (posteriormente, «La Bohemia de mi Tiempo») (Holguín, 1993, p. 140). Los primeros años de trayectoria literaria de Palma se desarrollaron durante un periodo de importantes cambios en el país y una gran y diversa producción intelectual peruana en poesía, literatura, teatro y ópera. Asimismo, Palma y sus amigos, como solían llamarse, fundaron en Lima, en setiembre del mismo año, el semanario político de oposición El Diablo, donde ve sus inicios en el periodismo político, teniendo a la sátira como su mejor arma para criticar a diversos personajes del gobierno, de la vida política y social del país. Así lo afirma Palma: «De 1848 a 1860 se desarrolló, en el Perú, la filoxera literaria, o sea pasión febril por la literatura» (Holguín, 1993, p. 140). Palma inicia así un largo recorrido por el mundo de las letras.

En 1851, el poeta, militar y político colombiano Julio Arboleda Pombo, exiliado forzosamente en el Perú, fue profesor de inglés de Palma. Fue, asimismo, uno de los escritores y poetas románticos más importantes de la Nueva Granada. "Arboleda tuvo una formación clásica, pero lo fascinaron los románticos españoles, franceses e ingleses, sobre todo Espronceda y Byron. [...] Arboleda representa con éxito el intento de armonizar y fundir el espíritu del conquistador con el del indígena, el alma de Europa con el alma de América» (EcuRed, 2019), aspectos que podrían haber influenciado positivamente en la manera en que Palma concibiera el idioma y la cultura inglesa, haciéndole despertar un especial interés por querer recorrer pronto estas grandes ciudades.

Durante este tiempo, la literatura tuvo importante transcendencia y aceptación. Sin embargo, si bien los vates gozaron del somero reconocimiento de los sectores altos y medios, también experimentaron la indiferencia, el desprecio y la desilusión. Lejos de ocultarlo, Palma 
denunció que el público era aristarco cuando presenciaba una obra local, pero era indulgente con las obras extranjeras, a las que consideraban magníficas:

Las bellezas pasan desapercibidas y solo resaltan a los ojos del zoilo los defectos e incorrecciones. Y después... allí está la imprenta. No escasearán artículos declarando, majistralmente [sic] y sin apelación, un estúpido al poeta y si se le honra mucho reconociendo algo de bueno en su trabajo, queda el recurso de afirmar que lo ha plajiado [sic]. - ¿De dónde?, pregunta sorprendido- Del infierno... de un tratadista alemán o turco, responde el criticastro con todo el aplomo que le proporciona su falta de pudor; y entre tanto el infeliz escritor dramático tiene que ahogar en germen sus facultades y romper su pluma; porque tal vez no le sobra energía para luchar contra la calumnia, la ignorancia y el ridículo (Holguín, 1994, p. VI).

Palma estrecha lazos de amistad y solidaridad con los bohemios, aunque también experimenta marcados factores que los separan e impiden su concatenación como el grupo influyente de aquel entonces: la edad de unos, la educación (provinciana y en el extranjero), la economía familiar y el roce social entre aristocráticos y los populares de sectores bajos. Este sinsabor, así como el retiro de los bohemios mayores y la necesidad de crecer y madurar en la creación literaria hizo que poco a poco dejara de hacerse literatura en el club de los bohemios y, finalmente, en 1860, llega el fin de esta época de vasta producción literaria, sentándose las bases para encaminarse, Palma y sus amigos, hacia la evolución de la producción literaria peruana.

\section{RICARDO PALMA EN AMÉRICA Y EUROPA}

Palma fue el privilegiado cronista y a la vez vocero de su grupo generacional. En 1860, a sus veintisiete años, Palma había experimentado la felicidad y el sufrimiento, y tenía la trayectoria y vocación de un escritor versado. Sin embargo, su rumbo cambiaría 
cuando, el 23 de noviembre de 1860, forma parte de un frustrado complot revolucionario dirigido por José Gálvez contra el presidente Castilla. Intensamente buscado por la fuerza pública, Palma solicita asilo político a Chile, se embarca el 20 de diciembre al país del Mapocho, donde escribe el poema «Navegando» (Palma, 1977, p. 460). En el destierro prosigue su actividad literaria. Palma debió atravesar por un periodo muy personal de experimentación que le dio fuerzas suficientes para madurar su propio estilo literario, buscando la perfección en sus escritos y publicando solo aquello que consideró un trabajo digno.

Aquí cabe resaltar la importancia de su buen conocimiento de inglés y francés, que le permite perfeccionarse en lo aprendido y leer no solo a numerosos autores clásicos y románticos, sino a escritores europeos no franceses vertidos a la lengua gala, como el viajero inglés Stevenson y los alemanes Samuel Pufendorf y Heinrich Heine (17971856). Heine fue una importante influencia para Palma. El poeta alemán era un enamorado de la libertad, de la Revolución francesa, fanático antimonárquico y adversario acérrimo de lo tradicional. Luchaba contra la autoridad política y literaria. Así emigró a París, que lo recibió con los brazos abiertos: había mucho de ático, y de parisién en él, por tanto, en su carácter y en su genio, impresionable, novelesco, sensible en el fondo, pero frívolo en la apariencia; y, a la vez, conservaba de su país natal una extraña mezcla de delicada ternura, de abstracción sutil y de quimérica fantasía, elementos contrapuestos, de cuyo choque nacía quizá el acervo sarcasmo que era la nota final de casi todas sus inspiraciones ${ }^{2}$. Palma encontró muy interesante a este autor, de quien tradujo varios poemas a partir de las versiones al francés de Gérard de Nerval ${ }^{3}$.

2 Prólogo de Teodoro Llorente a la obra Libro de los cantares de Heinrich Heine.

3 Se valió de la traducción realizada por el francés Gérard de Nerval (Poesías completas, p. 151, nota). 
Entre los poetas que mayor influencia debieron ejercer en Palma destacan Alphonse de Lamartine (1790-1869), George Sand (18041876), Alfred de Musset (1810-1857), Gérard de Nerval (1808-1855), Pierre-Jean de Béranger (1780-1857), Frédéric Soulié (1800-1847), Joseph Bouchardy (1810-1870). También se habría encontrado en Francia con Paul de Kock, notable novelista de la época ${ }^{4}$. Pero su preferido fue Victor Hugo (1802-1885), según propio testimonio, seguramente por sus altos ideales y el mensaje social transmitido en sus obras. En 1860 Palma publica en la Revista de Lima su primera versión de «La conciencia» (tomo V, 1860), fragmento de La leyenda de los siglos, dedicado a Pedro Ignacio Noboa, un buen conocedor de la literatura francesa y devoto de Victor Hugo. Entre los clásicos, Palma se identificó por completo con el influyente retórico y poeta Boileau (1636-1711).

En julio de 1864, Palma viaja a Europa como cónsul en el Pará (Brasil), donde se queda cerca de un año. Estuvo en Southampton un breve tiempo, donde pudo comprobar el alto grado de desarrollo de Inglaterra, la primera potencia mundial por entonces (Pérez, 2015, p. 150).

Luego Palma recorrió el Havre, París y Londres. En París conoce a Ascasubi, Gonçalves Dias, Torres Caicedo (Palma, 1977, p. 464) y se cree que a Alejandro Dumas padre. Todos ellos escritores románticos y de sentimientos nacionalistas. En Versalles, conoció al mariscal Andrés Santa Cruz, con quien pudo compartir una simpática anécdota personal que hoy es parte de la historia, tal como consta en su tradición «Una visita al mariscal Santa Cruz». En el Havre aparecen sus Pasionarias, cuyo prólogo de Luis Benjamín Cisneros afirma: «El lector encontrará, pues, en este libro, como habrá encontrado en las Armonías del mismo poeta, hermosos versos escritos bajo las

\footnotetext{
4 Riva-Agüero, «D. Ricardo Palma», p. 14. Quizá se trató de su hijo, Henri de Kock (1819-1892), también novelista popular.
} 
impresiones, siempre fogosas, del amor a la patria y a la libertad» (Palma, 1977, p. 468).

Palma también fue ferviente admirador de Walter Scott, quien cultivó la novela histórica en Inglaterra. Incluso, José de la RivaAgüero, que lo conocía desde muy joven, estableció la comparación entre Palma y Walter Scott (Gonzales, 2012, p. 250). Sin embargo, Palma hace la distinción entre «tradición» e historia así:

Menos estrechos y peligrosos son los límites de la Tradición. A ella, sobre una pequeña base de verdad, le es lícito edificar castillos. El tradicionista tiene que ser poeta y soñador. El historiador es un hombre del raciocinio y de las prosaicas realidades. La Tradición es la fina tela que dio vida a las bellísimas mentiras de la novela histórica, cultivada por Walter Scott en Inglaterra, por Alejandro Dumas en Francia y por Fernández y González en España (Forgues, 2013, p. 309).

Palma visitó Estados Unidos, probablemente entre comienzos y mediados de abril de 1865 (Pérez, 2015, p. 155), arribó al puerto de Nueva York a los treinta y dos años de edad. Según Holguín (2007) «la impresión que Nueva York le causó debió de ser muy fuerte, aunque no ha quedado consignada por escrito». Es posible que en Nueva York Palma conociera a Henry Wadsworth Longfellow, de quien tradujera una de sus más célebres composiciones: El salmo de la vida (The Psalm of Life), cuyo poema contiene una auténtica profesión de fe, de vivir activamente, sin lamentar el pasado ni subestimar el futuro, una forma de pensar que habría fascinado al tradicionista.

Por otro lado, Palma mantuvo una estrecha amistad con Nicanor Bolet Peraza, político, diplomático y escritor venezolano radicado en Nueva York, fundador de la Revista Mercantil y la Revista Ilustrada de Nueva York, para la que escribiría Palma. Más adelante, mantendría intactos los vínculos amicales con la cultura norteamericana gracias a 
la subvención que apoyó para su estimado amigo Julio César Tello en los Estados Unidos, hecho que lo acerca a Archer Milton Huntington, fundador de la Sociedad Hispánica de América.

Como hemos visto, Palma consolidó buenas relaciones literarias y personales con escritores del mundo occidental, por lo que cabe preguntarse en qué medida la lengua inglesa caló en su espíritu para lograr la excelencia de sus obras. Miguel Cané lo resume así:

El estilo de Ricardo Palma es su propiedad exclusiva e inimitable; pero aquel que, engañado por su pureza castiza, le supusiera una filiación únicamente española, sufriría un grave error. No se alcanza esta perfección sin conocer a fondo los humoristas ingleses, especialmente Swift y Henry Bayle; sin haber vivido en íntimo comercio con Molière, y entre los alemanes con Heine y Jean Paul. Indudablemente que sobre todos ellos está Cervantes; pero es precisamente el carácter de nuestra literatura americana la base ecléctica en que se apoya. Todo eso ha tomado su nota individual al pasar por el espíritu de Palma, dando por resultado ese estilo, lleno de chispa y malicia, que roza siempre los hombres y las costumbres sin cortar hasta el hueso; que no se desmiente jamás, manteniéndose en la atmósfera de picaresca ingenuidad que lo hace delicioso (Palma, 2000, párr. 28).

Es por ello que se cree que este primer contacto con importantes escritores de la cultura americana e inglesa constituyó una gran influencia en la composición de sus más célebres Tradiciones, así como un posible punto de partida de la inminente evolución intelectual plasmada en su poesía; para Palma, la poesía fue el pilar de las creaciones más bellas de su espíritu.

\section{CONCLUSIONES}

Como ya se ha señalado, Ricardo Palma fue un escritor nato. Desde muy pequeño demostró su gran afición por los idiomas, destacando en cuanto nivel se le impusiera. Está claro que para Palma la formación 
teórica de su disciplina no fue suficiente, pues el camino a la grandeza literaria pasó por agua tibia los conocimientos, e incidió en las vivencias, en las aproximaciones y en el estrecho contacto con aquel mundo que por entonces parecía conducir los hilos de la historia.

Cierto es que la literatura inglesa tuvo un papel destacado en la formación cultural de Palma, pues fueron numerosas las piezas literarias que desfilaron y se crearon bajo los confines de los territorios que lo vieron ir y venir y que impresionaron su fantasía durante la lectora juventud que le tocó vivir. Todas estas vivencias lo encumbraron más allá de nuestras fronteras, fundiendo con éxito el alma de Europa con el alma de América y el alma del Perú.

Palma fue un gran admirador y devoto de la literatura francesa clásica y romántica. En el área que nos ocupa, su faceta como traductor es de especial relevancia para estudiar la profusa determinación con la que buscó plasmar la belleza del alma de las letras, acometiendo con ímpetu la reproducción fiel de un mensaje de un idioma a otro, haciéndonos disfrutar de cada creación literaria extranjera en la tierra a la que amó con esa misma fidelidad.

Se cree que Palma encontró en los diferentes destinos del mundo occidental una cuna literaria que lo albergó con lo mejor de sus influencias. Como buen bohemio, Palma supo explorar de cerca la vida de las tertulias literarias, haciendo muchas amistades en el campo literario y entregándose a cuanta lectura pudiese, llenándose de la vasta experiencia de los grandes poetas e influyentes literatos americanos y europeos, quienes más adelante rindiesen pleitesía a los frutos de su pasión por las letras.

Según sus propias palabras, el tradicionista fue poeta y soñador, y desde sus primeros escritos, cargados de humor, romanticismo y saberes populares, el legado literario-histórico de Palma no dejó de pasar desapercibido, satisfaciendo al pensamiento que buscaba nuevos horizontes y al patriotismo que soñaba con nuevas glorias. 
La manera en la que Palma supo describir las costumbres de nuestro pasado de manera ágil, satírica y bien estructurada hace que un repaso por ciertos aspectos de la vida de este gran escritor resulte obligatorio si queremos desvelar los mensajes ocultos entre su pluma y sus páginas.

La estrecha relación que mantuvo Palma a lo largo de los años con la cultura anglosajona es un punto que podría acercarnos al verdadero espíritu de su pasión por las letras. Y para lograrlo debemos tener presente lo sentenciado por Heine (1885) sobre la traducción:

Es intento arriesgadísimo siempre, escribía, reproducir en prosa y en una lengua de procedencia latina, una obra métrica, compuesta en idioma de origen germánico. El pensamiento íntimo del original se evapora fácilmente en la traducción, y no queda más que algo parecido al resplandor de la luna disecado... ${ }^{5}$ (párr. 33).

¿Cómologró, entonces, nuestro notable escritor peruano, trascender en este encuentro cultural? Esta reflexión deja abierto el camino a los traductores apasionados como Palma, quienes, aficionados por la literatura, aún podemos escudriñar en el pensamiento íntimo de los escritores y salir airosos donde otros han tropezado.

\section{REFERENCIAS}

EcuRed (2019, 16 de abril). Julio Arboleda Pombo. https://www. ecured.cu/Julio_Arboleda_Pombo

Forgues, R. (2013). Claves ocultas del pensamiento de Palma. Aula Palma, (12), 307-320.

Gonzales, O. (2012). Ricardo Palma y el Novecientos. Retazos de reconstrucción epistolar. Yuyaykusun, (5), 245-260.

5 Prólogo de Heine a la traducción francesa de sus poesías, publicadas con el título de Poèmes et Légendes (París, 1855). 
Heine, H. (1885). Libro de los cantares. Prólogo de Teodoro Llorente. http://www.cervantesvirtual.com/obra-visor/libro-de-loscantares--0/html/ff0e47cc-82b1-11df-acc7-002185ce6064_2.htm

Holguín, O. (1993). Ricardo Palma y los bohemios: el grupo, cronología y guías. Boletín del Instituto Riva-Agüero, (20), 139-154. http://repositorio.pucp.edu.pe/index/bitstream/handle/123456789/113711/9670-Texto\%20del\%20art\%c3\%adculo-38258-1-10-20140729.pdf? sequence $=2 \&$ is Allowed $=y$

Holguín, O. (1994). Tiempos de infancia y bohemia. Ricardo Palma (1833-1860). Pontificia Universidad Católica del Perú, Fondo Editorial.

Holguín, O. (2007). Ricardo Palma y los Estados Unidos. http://www. cervantesvirtual.com/obra-visor/ricardo-palma-y-los-estadosunidos-0/html/017a6414-82b2-11df-acc7-002185ce6064_2.html

Nueva Acrópolis (2016). Poesía: El Salmo de la Vida. https://biblioteca. acropolis.org/poesia-salmo-la-vida/

Palma, R. (1977). Cien tradiciones peruanas. Prólogo, selección y cronología de José Miguel Oviedo. Biblioteca Ayacucho. https:// ibero2umich.files.wordpress.com/2011/10/cien-tradiciones.pdf

Palma, R. (2000). Tradiciones peruanas. Primera serie. http://www. cervantesvirtual.com/obra-visor/tradiciones-peruanas-primeraserie--0/html/ff170c4a-82b1-11df-acc7-002185ce6064_2.html

Pérez, C. (2015). Liberalismo criollo: Ricardo Palma, ideología y política (1833-1919). Universidad Ricardo Palma, Editorial Universitaria.

Segura, A. (1858). Teatro de Manuel A. Segura. https://archive.org/ details/bub_gb_C1ssAAAAIAAJ/page/n11

Wadsworth, H. (s. f.). A Psalm of Life. Poetry Foundation. https://www. poetryfoundation.org/poems/ 44644/a-psalm-of-life 